\title{
Aprendizaje situado en el diseño de entornos virtuales de aprendizaje: una experiencia de aprendizaje entre pares en una comunidad de práctica
}

\author{
Isabel Hevia Artime $\left({ }^{*}\right)$ y Aquilina Fueyo Gutiérrez $\left({ }^{*}\right)$ \\ ${ }^{*}$ ) Universidad de Oviedo - España
}

\section{RESUMEN}

El artículo analiza el potencial del aprendizaje situado entre pares en el contexto de una experiencia formativa con 155 estudiantes universitarios. Por un lado, se cuenta con un grupo de estudiantes de $4^{\circ}$ curso del Grado de Pedagogía que diseñan y ponen en marcha seis cursos MOOC. Por otro, está un grupo de estudiantes de $1^{\circ}$ curso que se matriculan en uno de esos MOOC y tienen como tarea la evaluación del diseño pedagógico de los mismos. A través de esta experiencia, se busca conocer qué beneficios reportan este tipo de experiencias tanto para el alumnado que enseña como para el que recibe la formación acerca de su campo profesional. Para ello, la recogida de información se realiza a través de la observación participante, el análisis documental y cuestionarios. Los resultados sugieren que, a través de esta modalidad de aprendizaje situado entre pares, los estudiantes desarrollan un aprendizaje de más profundidad evidenciándose indicadores del desarrollo de competencias profesionales de gran interés, entre las que destacan las relacionadas con el pensamiento reflexivo y las vinculadas al desempeño profesional y las competencias digitales.

Palabras Clave: comunidad de práctica, aprendizaje entre pares, enseñanza situada, entornos virtuales, innovación educativa.

\section{Situated Learning in the design of virtual learning environments: an experience of peer learning in a com- munity of practice}

\section{ABSTRACT}

This article analyzes the potential of peer-to-peer situated learning in the context of a formative experience with 155 university students. On the one hand, we have a group of 4 th year students of the Degree in Pedagogy who design and implement six MOOC courses. On the other hand, we have a group of 1st year students who enroll in one of those MOOCs and their tasks of the evaluation of the pedagogical design of the MOOC they chose. The aim of this experience is getting to know what benefits are obtained by the students who teach and by those who receive training about their professional field. For that purpose, data collection is done through participants observation, documentary analysis and questionnaires. The results suggest that, through this modality of peer-to-peer learning, students develop more in-depth learning which revealing indicators of the development of professional competencies of great interest, among them can be highlighted those related to reflective thinking and digital skills and performance.

Keywords: community of practice, peer-to-peer learning, situated teaching, virtual environments, educational innovation.

\section{Introducción}

Actualmente nos encontramos en un contexto universitario donde las necesidades emergentes de la sociedad, fruto de los grandes cambios sociales y tecnológicos de los últimos tiempos, hace que el dominio de los contenidos científicos por parte de los docentes no sea suficiente para facilitar la construcción de conocimientos (Gisbert y Esteve, 2011). Las maneras en que los estudiantes acceden y procesan la información han cambiado por el uso de dispositivos digitales y el acceso a una información transmedia, el aprendizaje se ha vuelto más colectivo y la negociación de contenidos adquiere un papel relevante. Se habla de una sociedad donde hay una diseminación del conocimiento, un aprendizaje a lo largo de toda la vida que se enriquece a través de la experiencia social en nuevos entornos digitales. La educación superior, gracias a las nuevas tecnologías, ha acelerado el proceso de globalización de la educación y se encuentra en constante evolución (McDonald y Cater-Steel, 2016). Se requiere, por 
tanto, formar ciudadanos con capacidades para la innovación, la imaginación, la creación y la posibilidad de interactuar y diseñar proyectos compartidos con cualquier persona, en cualquier lugar y momento (Fernández y Anguita, 2015). Esta cuestión evidencia la necesidad de vincular los procesos de aprendizaje individual y aprendizaje colectivo (Barrera-Corominas, Fernández-de-Álava y Gairín, 2014) con la creación de nuevos significados y las oportunidades de interactuar con distintas fuentes o contextos, lo que choca con el modelo imperante en las instituciones de educación superior anclado fuertemente en la transmisión unidireccional de contenidos (Hernández-Selles, González-Sanmamed y Muñoz-Carril, 2015).

En este escenario, donde el aprendizaje a través de la práctica y de las interacciones sociales constantes se vuelve necesario, se presentan algunos de los resultados del estudio realizado sobre el diseño de entornos virtuales de formación por parte de futuros profesionales de la educación. La experiencia se apoya en un modelo de aprendizaje en red basado en las comunidades de práctica y la cognición situada, donde el alumnado pueda alternar su rol como aprendiz con el de contribuidor a la construcción activa de conocimientos a fin de resolver problemas de forma creativa y con distintos enfoques, desde una perspectiva multidisciplinar (Fischer, Rohde y Wulf, 2007). En este caso, el problema a resolver es la creación de un curso MOOC por parte de unos estudiantes inexpertos con la finalidad de ofertarlo a sus compañeros de cursos inferiores que, a su vez, deben evaluar el diseño pedagógico de esos cursos.

Se adopta, por tanto, la idea de una universidad entre pares defendida por Cobo y Moravec (2011) que ofrezca modelos de enseñanza colectiva y entre iguales. El aprendizaje entre pares es definido por Boud et al. (2001) como aquel que se genera en situaciones educativas en las que los estudiantes tienen oportunidades recíprocas de aprender y enseñar; de aprender de y con sus propios compañeros. Este tipo de enseñanza favorece el aprendizaje mutuo e interdependiente, así como una apertura del conocimiento que facilita la transferencia horizontal, lo que Cobo y Movarec (2011) denominan aprendizajes invisibles. Se estaría haciendo referencia a aquellos aprendizajes informales construidos por los propios estudiantes fuera del aula, estimulados por las nuevas tecnologías y que son invisibles al sistema educativo, pero con los que resulta imprescindible dialogar desde la educación formal. El adjetivo invisible se asienta en un modelo de educación informal soportado por comunidades de aprendizaje, subrayando la importancia del aprendizaje que se produce fuera de las relaciones, espacios y tiempos puramente académicos (Enguita, 2013). Desarrollar una actividad formativa basada en los principios del aprendizaje invisible nos permite comprobar si es posible socializar a un grupo de estudiantes en contextos de intercambio colaborativo y trabajo semiautónomo, desarrollando esas competencias invisibles que luego podrán emplear en su ámbito profesional. Uno de los principales atractivos de este aprendizaje es la construcción de un modelo educativo basado en comunidades de aprendizaje en donde se regula el conocimiento que se construye y se comparte (Cobo y Moravec, 2011). Todo parece indicar, por tanto, que la colaboración mediante comunidades va a tener un papel relevante para que los estudiantes hagan suyo el conocimiento y le den un significado, involucrándose activa y socialmente en su construcción.

Al hablar de comunidad de aprendizaje es inevitable hacer referencia al concepto de comunidad de práctica propuesto por Wenger (2001) quien la define como un grupo de personas que se involucran en un aprendizaje colectivo, comparten una preocupación o pasión por algo que hacen y aprenden cómo hacerlo mejor a medida que interactúan regularmente. En la comunidad universitaria la teoría de las comunidades de práctica ha sido aplicada en diferentes niveles y contextos con resultados exitosos tanto a nivel individual como organizativo (Gil-Izquierdo, Fernández-Pinedo y Pérez-Encinas, 2017) evidenciándose que no existe una única manera de afrontar la creación de comunidades de práctica (Davies et. al, 2017). McDonald (Reaburn y McDonald, 2016) fue pionera en organizar una comunidad de práctica piloto en una universidad de Australia y a ella le siguieron experiencias como las compiladas en la obra de McDonald y Cater-Steel (2016) donde nos ofrecen ideas sobre estrategias de implementación de los fundamentos teóricos de las comunidades de práctica en la educación superior.

La organización del alumnado como una comunidad de práctica se hace para darle la oportunidad de participar en una actividad conjunta en donde todas las personas que integran esa agrupación podrán experimentar la propia práctica con significatividad. Así, en función de lo significativo que resulte ese aprendizaje o del tipo de interacciones colaborativas que propicie, podrá transferirse a otras situaciones distintas a las originales (Díaz-Barriga, 2006). Se entiende que el conocimiento es parte y producto de la actividad, del contexto y de la cultura en que se desarrolla (Brown, Collins y Duguid, 1989). Se habla, por tanto, de un aprendizaje situado que toma como punto de partida los estudios de Vygotsky (1986) y de autores como Bereiter (1997), Lave (1997), Lave y Wenger (1991) o Wenger (2001), y en donde se afirma que el conocimiento tiene lugar en un contexto y situación concreta, siendo resultado de la acción de la persona que aprende en interacción con otras personas (Díaz-Barriga, 2003). Por tanto, aprender y hacer son acciones inseparables y la enseñanza se debe centrar en prácticas educativas auténticas que no se abstraigan de las situaciones en las que se aprende y enseña (Díaz-Barriga, 2003). Según este concepto, el aprendizaje es el desarrollo de aprendices a expertos en donde la oportunidad de participación en una actividad significativa, el derecho a la pertenencia y la opción a espacios de práctica y experiencia son más importantes que el aula universitaria, sus docentes o los propios materiales de aprendizaje (Niemeyer, 2006). Diferentes investigaciones (Barrio y García, 2011; González y García, 2012; Leguizamo, Montaño y Villarroel, 2004) han puesto de manifiesto que, en el contexto universitario, el aprendizaje situado facilita la adquisición de competencias profesionales al suponer un aprendizaje activo que posibilita que los estudiantes puedan tomar decisiones por sí mismos, delimitadas a acciones que faciliten aprender a aplicar (Paz, 2007).

\section{Metodología y desarrollo de la investigación}

Se ha llevado a cabo un estudio de caso que aborda distintas estrategias de recogida de información a través de la complementariedad metodológica. Para ello, se realiza un estudio de carácter interpretativo que implica un análisis en profundidad de una experiencia con el fin de aportar información relevante para la interpretación y teorización en base al caso de estudio. El punto de partida es una experiencia educativa fundamentada en una estrategia de enseñanza situada donde el alumnado tiene la oportunidad de ejercer como profesional, así como afianzar los conocimientos adquiridos a través del aprendizaje colectivo con sus iguales. La idea de dar seguimiento al desarrollo de esta actividad está en recabar información que permita dar respuesta a las siguientes hipótesis:

1. Una experiencia educativa planteada desde las premisas del aprendizaje situado ayuda a desarrollar en el alumna- 
do habilidades y competencias profesionales de comunicación, planificación, organización, toma de decisiones y autoaprendizaje.

2. En contextos de enseñanza universitaria el desarrollo de experiencias entre pares mediante comunidades de práctica reporta beneficios tanto para el alumnado que enseña, como para aquel alumnado más novel que recibe la formación sobre su campo de conocimiento y puede reflexionar de manera activa sobre las metodologías que posibilitan sus aprendizajes.

\subsection{Contexto}

La experiencia estudiada se enmarca en un proyecto de innovación interdisciplinar de la Universidad de Oviedo denominado "Diseñando MOOCs. Una experiencia profesionalizadora y de tutoría entre estudiantes universitarios" (PAINN-16-038) y que tiene lugar en el Grado de Pedagogía. Dicho proyecto se asienta en las bases del Proyecto ECO E-learning, Communication and Open Data: Massive Mobile, Ubiquitous and Open Learning de la Comunidad Europea, centrado en el diseño y desarrollo de innovaciones de la metodología y las herramientas tecnológicas que se utilizan para los cursos MOOC (Osuna-Acedo, Marta-Lazo y FrauMeigs, 2018).

En esta experiencia se opta por una metodología de trabajo en donde el alumnado tiene que diseñar un curso en la modalidad de enseñanza virtual con las características de un MOOC y su posterior ejecución. Para ello, se crea una comunidad de práctica en donde el alumnado de $4^{\circ}$ curso trabaja en el diseño de 6 MOOC y asume el rol de e-teachers durante 4 semanas. Los destinatarios de estos MOOC es el alumnado de $1^{\circ}$ curso que, a su vez, realiza la evaluación del diseño pedagógico de los mismos, fomentando así el aprendizaje entre iguales. Para ello, cuentan con un grupo de expertos universitarios que tienen el papel evaluadores y asesores en la fase de diseño de los MOOC y evaluación final. El desarrollo de los MOOC se lleva a cabo en la plataforma Open Mooc del Proyecto ECO Learning, lo que posibilita que los cursos estén disponibles en abierto.

La experiencia se ha desarrollado en cinco fases: Una primera de formación en el diseño de entornos virtuales del alumnado de $4^{\circ}$ curso, donde se determinan las temáticas de los MOOC, surgidas de las demandas del alumnado de $1^{0}$ curso. Los temas son: redes sociales, igualdad de género, ciberacoso, prevención de drogodependencias, intervenciones antidiscriminación y estereotipos de género en la publicidad. La segunda fase comprende el diseño pedagógico de los MOOC, que debe cumplir con unos requisitos mínimos propios de esta modalidad formativa y acordados entre todos los participantes. A partir de este momento, se trabaja en un entorno colaborativo online compartido con los docentes-expertos que les asesoran durante todo el proceso. La tercera fase comprende tareas de difusión y comunicación. Debido a que los MOOC estarán en abierto, podrán ser cursados por cualquier persona interesada. En la cuarta fase tiene lugar el desarrollo en abierto de los cursos (del 24 de octubre al 20 de noviembre de 2016). Por último, hay una quinta fase de evaluación que implica: una autoevaluación grupal e informe individual (alumnado $4^{\circ}$ curso) y un cuestionario de valoración y análisis pedagógico grupal (alumnado $1^{\circ}$ curso).

\subsection{Participantes}

En esta experiencia han participado 155 estudiantes del Grado de Pedagogía (98 de $1^{\text {o }}$ curso y 57 de $4^{\text {o }}$ curso) de la Universidad de Oviedo que cursan las asignaturas de "Entornos Virtuales para la Educación y la Formación" ( $4^{\mathrm{O}}$ curso) y "Didáctica General" ( $1^{\circ}$ curso). Además del alumnado, se contó con un grupo de 5 profesores universitarios que asumieron el rol de "expertos" supervisando los MOOC durante todo el proceso. Por otro lado, hay que destacar que los MOOC diseñados estuvieron abiertos a la participación externa, alcanzando un total de 536 personas matriculadas y cuya distribución viene recogida en la Tabla 1:

Tabla 1. MOOC diseñados y participantes

$\begin{array}{lccc}\text { MOOC } & \text { № e-teachers } & \text { № estudiantes } & \text { \% finalización } \\ \text { Curso 1 } & 9 & 90 & 14 \% \\ \text { Curso 2 } & 11 & 63 & 7 \% \\ \text { Curso 3 } & 9 & 89 & 19 \% \\ \text { Curso 4 } & 6 & 102 & 15 \% \\ \text { Curso 5 } & 11 & 81 & 3 \% \\ \text { Curso 6 } & 11 & 111 & 13 \%\end{array}$

Se evidencia que los cursos han despertado interés, más allá del alumnado universitario para el que iban dirigidos, siendo el número de personas que se matricula en los mismos, superior al inicialmente previsto. En cuanto al porcentaje de finalización, tal como es sabido, los MOOC tienen altas tasas de deserción (Bartolomé y Steffens, 2015). La mayoría reportan unas tasas de finalización de menos de $10 \%$ y un promedio de alrededor del 7\% (Jordan, 2014). Así la tasa de abandonos de MOOC impartidos por Stanford, Massachusetts Institute of Technology (MIT) y la UC Berkeley es entre el $80 \%$ y el 95\% del total del alumnado (Daniel, 2012). En el caso estudiado puede decirse que, a excepción de dos cursos en donde el porcentaje de finalización es inferior al 10\%, el resto alcanzan unas tasas de finalización aceptables, especialmente el Curso 3 que se sitúa cerca del 20\%. Podría pensarse que estos porcentajes, en cierta medida, están desvirtuados por el alumnado de $1^{\underline{o}}$ curso que se matricula en los MOOC, sin embargo, este alumnado sólo supone entre un 15\% $-18 \%$ del alumnado que se matricula en el total de los seis cursos, no estando obligados a cursar el MOOC hasta el final.

\subsection{Instrumentos}

En este estudio se han seguido algunas de las estrategias propuestas por Benavides, Madrigal y Quiroz (2009) para evaluar los resultados de un programa de enseñanza situada: observación participante del proceso, reuniones con los participantes, autoevaluaciones, cuestionarios y análisis de documentación. En la Tabla 2 se recoge la relación de los elementos a analizar y las fuentes de datos a las que se ha recurrido: 
Tabla 2. Fuentes de información, codificación y su relación con los elementos de análisis

Aspectos a analizar

Seguimiento experiencia
№

6

9 semanas

1

6

57

95

24

6
Códigos

EVC

OBP

CDP

CG4

II4

CI1

IG1

MOC
La observación participante tiene lugar durante las 9 semanas que dura la experiencia formativa y que abarca tanto el diseño como la puesta en práctica de los MOOC. Esta observación es llevada a cabo por los cinco docentes implicados en el proyecto, estableciendo puntos intermedios de reflexión para valorar el desarrollo de la experiencia. Además, el seguimiento del diseño de cada curso se lleva a cabo a través de un espacio virtual colaborativo compartido por docentes-expertos y alumnado de $4^{\underline{0}}$ curso, lo que permite analizar cómo los estudiantes van configurando su comunidad de práctica a la vez que desarrollan su propio currículo de aprendizaje.

Por otro lado, se emplean dos tipos de cuestionarios online: una autoevaluación grupal (alumnado $4^{\circ}$ curso), compuesta por 10 ítems cerrados y 5 abiertos, y un cuestionario individual (estudiantes $1^{\circ}$ curso) constituido por 30 ítems de respuesta cerrada, en donde tienen que valorar el diseño pedagógico de los MOOC cursados. Este último es complementado con unas fichas que de manera grupal cubren los estudiantes de $1^{\underline{0}}$ curso en donde ofrecen aportaciones de tipo cualitativo sobre el diseño pedagógico de los MOOC. Este análisis documental se completa con los informes individuales realizados por los estudiantes de $4^{\underline{0}}$ curso, así como la revisión de los materiales producidos durante toda la experiencia formativa.

El estudio de la información cualitativa recabada se realiza a partir del método de análisis de contenido (Bardin, 1986), consistente en un proceso de categorización mixta en la que se parte de categorías que tienen una significación relevante en relación con los propósitos del estudio. Por otro lado, para el análisis de los datos recogidos a través de los cuestionarios, se ha empleado el paquete estadístico IBM SPSS versión 22 para Windows a través del cual se ha llevado a cabo un análisis descriptivo de frecuencias.

\section{Resultados}

Tras analizar en profundidad la información recogida, se procede a sintetizar algunos de los resultados más relevantes en base a las dos hipótesis que guían nuestro trabajo. Para apoyar el discurso, se incorporan fragmentos de las narrativas de los propios estudiantes, así como datos extraídos de los cuestionarios.

\subsection{A la búsqueda de altos niveles de aprendizaje (alumnado $4^{o}$ curso).}

Cuando se habla de aprendizaje de "alto nivel" se está haciendo referencia a aprendizajes transformadores que se apoyan en la teoría constructivista e implica una reinterpretación de co- nocimientos previos, así como aprehender el propio modo de gestionar y actuar a partir de los propios valores (Mezirow, 2000; Taylor, 2008). Esta orientación transformativa también aspira a producir una verdadera catarsis en nuestro alumnado, no es sólo que acumule e integre con armonía los nuevos aprendizajes con los previos, sino que sepa someter a crítica todo lo adquirido y lo que está por adquirir (Cela-Ranilla, et al., 2015; Fueyo y Hevia, 2017). Es por ello por lo que las narrativas de los estudiantes de $4^{\circ}$ curso resultan sumamente interesantes para determinar la consecución de esos aprendizajes a través de la experiencia en la que participan. Así, entre los aspectos que más enfatizan los estudiantes en sus informes está la gran responsabilidad que tuvieron que asumir en esta práctica, algo a lo que no están acostumbrados y que les ha generado cierta inseguridad, al tener que dar una respuesta real a las demandas de sus aprendices:

Veíamos que no solo enseñamos nuestro tema a alumnos inexpertos en la materia, sino que también enseñamos a profesionales que puedan ampliar sus conocimientos del propio tema del MOOC (AI4-13).

En relación con esta cuestión, Lave y Wenger (1991) realzan la participación, la interacción y el compromiso de los estudiantes dentro de este tipo de experiencias como características esenciales en la construcción consciente del conocimiento. En nuestro caso, ese compromiso se identifica a través de los relatos en donde los estudiantes evidencian el modelo pedagógico que intentaron construir, su ideal educativo que han pretendido poner en práctica a través de los MOOC diseñados, fruto de la reflexión sobre la teoría y de sus propias vivencias como estudiantes:

Mucha gente vemos una especial importancia a que los procesos de enseñanza-aprendizaje se conviertan en experiencias vivenciales, que dejen de producir aprendizajes superficiales y ajenos al individuo, es decir, buscamos una implicación real y efectiva, una conexión común en el proceso educativo (AI4-10).

Desde un principio hemos querido enfocar el MOOC como un curso que no descontextualizase la teoría, sino que el alumnado viese claramente el nexo con la práctica y qué haría de encontrarse en una situación en concreto relacionada con nuestro tema (AI4-41).

Por otro lado, al enfrentarse a situaciones reales, el propio alumnado cuestiona algunos aspectos que, previsiblemente, ante otros trabajos académicos más tradicionales no se plantearía. Tal es el caso de la reflexión suscitada acerca del papel que van a asumir como docentes y el papel que esperan promover entre sus estudiantes. De esta decisión depende buena parte de la planificación de las actividades del curso y su orientación metodológica. Podría decirse que, sobre esta cuestión, 
ha habido una unanimidad en los seis cursos en donde se ha optado por un modelo en donde se promueva la participación del alumnado y donde los docentes sean meros mediadores de conocimiento:

El papel que pretendiamos que interpretaran nuestros estudiantes era de crítica frente a los materiales que les estábamos presentando, que reflexionaran sobre diversos aspectos en relación con los mismos para que pudieran darse cuenta de la necesidad de tomar medidas para cambiar esa realidad social tan cercana. (AI4-41).

Aunque los profesores y profesoras fuesen en un principio quienes proporcionaban los materiales y realizaban la función formativa, rápidamente el alumnado, gracias a los espacios de participación creados y dinamizados por parte del equipo docente, comenzó a ejercer un rol activo en el proceso de enseñanza-aprendizaje; proponiendo lecturas, haciendo evidentes sus intereses e inclinaciones -ante las cuales se les recomendaban libros o páginas web-, participando en debates entre discentes y docentes... (AI4-10).

Otro aspecto que destacar, muy presente en todo el proceso de análisis, tiene que ver con la interacción social como elemento fundamental a la hora de favorecer los aprendizajes en un curso online, con la idea de promover un cambio conceptual a través del intercambio de información entre compañeros que tienen diferentes niveles de conocimiento sobre el tema. Es por ello por lo que los estudiantes de $4^{\circ}$ curso dan gran importancia a la idea de crear una comunidad, en donde se promueva no solo la interacción, sino la motivación por seguir adelante con el curso:

Hemos intentado crear un espacio de grupo, de comunidad donde todos formábamos parte y todos nos ayudábamos a crear conocimiento. Se podría decir que los foros de la plataforma y que, más concretamente, las comunidades de Google+ se asemejan a las comunidades de aprendizaje colaborativo de las que habla Nicholas Burbules (AI4-40).

Lo primero que los docentes del curso llevamos a cabo es crear un entorno positivo y agradable en el que se fomente la calidad de las interacciones y en el que promovamos la participación de un número considerable de estudiantes con el fin de crear un contexto educativo con presencia social suficiente, que sea coherente con los contenidos y que impulse los aspectos relacionados con la presencia cognitiva (AI4-14).

En cuanto a los aprendizajes adquiridos, son numerosos. El integrar la práctica a la vida real, a través de situaciones específicas con las que un profesor de e-learning se enfrenta en el día a día, les ha permitido avanzar en aprendizajes que han puesto en acción durante las cuatro semanas que los cursos estuvieron en línea:

Ha resultado muy enriquecedor compartir experiencias sobre las distintas formas de enfocar los temas a tratar y poder llegar a acuerdos, poder ponerse en una perspectiva distinta a la que se tiene habitualmente, gracias a la transferencia y comunicación con otras personas (AI4-2).

Nosotras, para desarrollar el curso, hemos tenido que identificar las tecnologías necesarias y las herramientas que promueven el in- tercambio de ideas entre los alumnos y las alumnas mediante la red y la educación interconectada. (AI4-14).

A lo largo de la experiencia, también se han identificado una serie de disfunciones que tienen que ver, principalmente, con los tiempos disponibles para el desarrollo de la asignatura en la que tiene lugar la experiencia. La condensación de clases teóricas y prácticas en un corto periodo de tiempo ( 9 semanas) hace que el ritmo de trabajo sea muy intenso y los estudiantes no están acostumbrados, además de que cursan otras asignaturas al mismo tiempo: "los plazos son demasiado cortos, lo que conlleva estrés y presión" (CG4-1). Por otro lado, se han puesto de manifiesto dificultades internas de los grupos debidas al número de integrantes, más numerosos de lo habitual, lo que ha originado alguna tensión: "éramos demasiadas personas para el trabajo grupal y es difícil valorar lo que cada miembro del grupo realiza e intentar que otros compañeros cumplan con su tarea" (CG4-4).

Entre los aspectos que más inseguridad han generado a los estudiantes, y donde mayores dificultades han tenido, ha estado en la moderación de los foros de sus cursos, en donde tenían que enfrentarse con estudiantes reales, ellos mismos admiten que esta ha sido la parte más difícil de la experiencia:

Como docentes, nuestra intervención es esencial, tanto en actividades individuales como aquellas que se desarrollan en los foros. Ha sido todo un reto para nosotras y nosotros tener habilidad para comunicarnos a través de la escritura (AI4-14).

En mi opinión, la parte que más difícil nos ha resultado ha sido el realizar un feedback continuo en los comentarios del foro, pues debido a los horarios y los otros trabajos de otras asignaturas de la carrera nos ha sido complicado dedicar el tiempo que considerábamos necesario a esta tarea en concreto (AI4-18).

A través de estos relatos, se observa como los estudiantes de $4^{\underline{o}}$ curso han llegado a considerarse verdaderos e-teachers, se refieren a ellos mismos como docentes y realizan reflexiones y planteamientos desde su nuevo rol.

3.2. Aprendizaje entre iguales: el cambio de roles y posiciones (alumnado $1^{\underline{o}}$ curso)

Teniendo en cuenta los planteamientos del aprendizaje situado referidos a los procesos de participación, en relación con el alumnado de $1^{\circ}$ curso que interviene en la experiencia, la cuestión que debería formularse es ¿qué aprenden estos estudiantes? Es cierto que las temáticas de los MOOC son muy variadas, pero la finalidad última no es solo un aprendizaje de los contenidos de esos MOOC, sino además conseguir que aborden su análisis pedagógico y que puedan utilizar en ese análisis los contenidos vistos en la asignatura de Didáctica General. El cuestionario final que se pasa a estos estudiantes arroja algunos resultados que nos permiten formular ideas acerca de la valoración pedagógica que realizan de los MOOC y sus aprendizajes entre iguales. Algunos de esos resultados pueden verse reflejados en la Tabla 3.

Tabla 3. Opiniones sobre los MOOC cursados (alumnado $1^{\circ}$ curso)

En tu opinión, hasta qué punto el curso que has realizado...

Está diseñado para alcanzar los objetivos propuestos

Fomenta la discusión y la reflexión personal en el campo trabajado

Promueve la implicación del estudiante en el curso

Promueve la interacción entre estudiantes

\section{Completamente En gran medida En alguna media}

Inadecuadamente

No lo sé

$\begin{array}{lllll}23,21 \% & 48,21 \% & 21,43 \% & 5,36 \% & 1,79 \% \\ 30,36 \% & 46,43 \% & 21,43 \% & 1,79 \% & 0,00 \% \\ 19,64 \% & 51,79 \% & 25,00 \% & 3,57 \% & 0,00 \% \\ 30,36 \% & 30,36 \% & 30,36 \% & 7,14 \% & 1,79 \%\end{array}$

Aula Abierta, volumen 47, nº 3, julio-septiembre, 2018, págs. 347-354 
Se observa que un número importante de los estudiantes (71,42\%) consideran que los MOOC están completamente o en gran medida diseñados para alcanzar los objetivos propuestos. Además, un porcentaje similar $(76,79 \%)$ valora que el planteamiento de los cursos favorece completamente o en gran medida la reflexión sobre los temas abordados, así como la implicación del alumnado en los mismos (71,43\%). Esto mismo se recoge en los informes grupales analizados:

Los mensajes que intentan transmitir son positivos y la forma en la que nos lo enseñan es entretenida, ya que no tenemos que aprender de forma memorística los contenidos, sino que se basan más en la experiencia y aprendemos haciendo, algo que autores pedagógicos como Freinet ya fomentaban (IG1-1).

$\mathrm{El}$ aspecto en donde no parece haber un consenso es el referido a la promoción de la interacción entre los estudiantes dentro del curso, aunque, en líneas generales, parecen valorar que sí se ha promovido.

Entrando en cuestiones más didácticas, la Tabla 4 nos muestra algunas de las valoraciones realizadas por el alumnado de $1^{\mathrm{O}}$ curso en relación con las actividades y recursos diseñados en los diferentes MOOC.

Tabla 4. Opiniones sobre las tareas y materiales de los MOOC cursados (alumnado $1^{\circ}$ curso)

\section{TAREAS Y MATERIALES}

Distribución de la carga de trabajo durante el curso

Diseño de tareas individuales

Diseño de tareas colaborativas

Videos y video lecciones

Documentos proporcionados

Materiales audiovisuales ofrecidos

Respuestas dadas por el equipo docente (en foros, chats...)

Usabilidad de la plataforma

Adecuación de los quiz, juegos y tests online

$\begin{array}{cccccc}\text { Muy buena } & \text { Buena } & \text { Adecuada } & \text { Pobre } & \text { Muy pobre } & \text { No lo se } \\ 14,29 \% & 42,86 \% & 33,93 \% & 7,14 \% & 1,79 \% & 0,00 \% \\ 19,64 \% & 32,14 \% & 35,71 \% & 10,71 \% & 0,00 \% & 1,79 \% \\ 16,07 \% & 35,71 \% & 37,50 \% & 7,14 \% & 0,00 \% & 3,57 \% \\ 28,57 \% & 23,21 \% & 25,00 \% & 16,07 \% & 1,79 \% & 5,36 \% \\ 30,36 \% & 33,93 \% & 33,93 \% & 1,79 \% & 0,00 \% & 0,00 \% \\ 30,36 \% & 33,93 \% & 23,21 \% & 10,71 \% & 0,00 \% & 1,79 \% \\ 23,21 \% & 30,36 \% & 25,00 \% & 14,29 \% & 1,79 \% & 5,36 \% \\ 19,64 \% & 28,57 \% & 37,50 \% & 10,71 \% & 0,00 \% & 3,57 \% \\ 30,36 \% & 21,43 \% & 33,93 \% & 5,36 \% & 1,79 \% & 7,14 \%\end{array}$

En líneas generales, hay una opinión favorable a que la distribución de la carga de trabajo durante las semanas en las que tiene lugar el curso ha sido buena o muy buena $(57,15 \%)$. En cuanto a las actividades, tanto las individuales (51,78\%) como las colaborativas $(51,76 \%)$ se consideran buenas y muy buenas, teniendo una valoración un poco más baja las individuales $(10,71 \%$ las considera pobres). En relación con los videos y lecciones diseñados, algo más de la mitad (51,78\%) los consideran buenos o muy buenos mientras que uno de cada cuatro estudiantes los considera adecuados. En el resto de materiales diseñados para el curso, las respuestas son variadas, oscilando entre muy buenas $\mathrm{y}$ adecuadas.

Anteriormente se veía como una de las preocupaciones de los estudiantes de $4^{\mathrm{o}}$ curso era la interacción en los foros, algo que les supuso todo un reto. Sobre este aspecto, el alumnado de $1^{\underline{0}}$ curso da unas valoraciones más bajas que en otros ítems, de hecho, un 14,29\% considera que estas interacciones han sido pobres, tal como se recoge en alguno de los informes grupales analizados:

Creemos que un aspecto que se podría mejorar sería la interacción entre los alumnos y profesores, es decir, hay veces que hemos puesto algún comentario en el foro o en la misma página del MOOC y no hemos tenido respuesta y creemos que este es un aspecto que se debe mejorar (IG1-10).

Por otro lado, otros datos que nos arroja el cuestionario es que un $87,04 \%$ considera que a través del MOOC realizado ha aprendido y, además, un $74,07 \%$ piensa que esos conocimientos tienen aplicación para su vida profesional, valorando muy positivamente los contenidos diseñados y trasmitidos por sus compañeros de $4^{\circ}$ curso:

Todos estamos de acuerdo que volveríamos a realizar estos MOOC, dado que nos han ayudado a ampliar nuestras perspectivas de la vida. Nos parece muy importante informarnos de estos temas, aunque son poco valorados por las instituciones educativas formales, porque, en definitiva, son temas cuyo conocimiento nos van a ayudar a vivir de una manera más consciente y feliz. (IG1-3). Por último, se ha querido concluir con una cita de una estudiante de $1^{\circ}$ curso que evidencia que uno de los factores más positivos de la enseñanza entre iguales es la motivación:

Además, el saber que este curso lo han preparado alumnos de cuarto de carrera de Pedagogía me supone una motivación extra para seguir en la carrera, ya que me parece una actividad muy llamativa y que me encantaría cuando llegue a cuarto ser capaz de diseñar yo misma un curso online para contribuir a la construcción de una sociedad mejor (IG1-14).

La participación en auténticos espacios de aprendizaje compartido entre estudiantes de diferentes niveles parece ser el motor que impulsa la transferencia de conocimientos, en donde todos aprenden. Esto encuentra correspondencia con lo que plantea Lave (1997) acerca de los aprendices novatos (en nuestro caso, alumnado de $1^{\circ}$ curso) que gradualmente van manejando los conceptos sobre Didáctica General, aprendiendo a pensar y a interactuar con ellos e incluso a cuestionarlos, incrementando así sus conocimientos.

\section{Conclusiones}

Los resultados obtenidos ponen de manifiesto que una metodología que favorezca el aprendizaje situado destinado a desarrollar competencias y habilidades profesionales de comunicación, planificación y organización tiene como consecuencia un aprendizaje profundo por parte del alumnado. Se considera que, con este enfoque, se movilizan los siguientes elementos:

1. La responsabilidad ante las demandas reales de otros estudiantes que participan en la experiencia. Una responsabilidad que obliga a resituar el papel de aprendiz y a 
reformularlo cuando asume funciones de diseño, planificación, mediación y organización. Funciones propias de la profesión para la que se está formando que se ponen en juego resolviendo problemas de forma creativa y con distintos enfoques, desde una perspectiva multidisciplinar, en línea con lo señalado por Fischer, et al. (2007), pero también de forma innovadora, inteligente y colaborativa con personas de su mismo perfil, profesionales expertos y aprendices con una experiencia menor.

2. Una reconstrucción más consciente y activa del contenido de la asignatura de estudio que se visibiliza cuando el alumnado pone de manifiesto el esfuerzo por crear una práctica que sea coherente con sus ideales educativos, integrando en su diseño la "teoría" pero también sus vivencias y experiencias prácticas como estudiantes, es decir, la puesta en práctica de los aprendizajes invisibles definidos por Cobo y Moravec (2011).

3. El hecho de que la comunidad de práctica creada someta a discusión su conocimiento y lo ponga a funcionar en una situación concreta solo es posible cuando el aprendizaje se realiza a través de la práctica y las interacciones sociales son constantes, cuando se desarrolla la autonomía en el aprendizaje (Rodríguez, 2006).

4. La interacción con el papel de las personas expertas que evalúan, asesoran y dan mayor peso a la situación real que se aborda, obliga al grupo-comunidad a afrontar sus demandas y darles respuestas adecuadas.

5. Esta experiencia supone un punto de inflexión frente a metodologías más tradicionales en las que el alumnado no tiene la exigencia de reflexionar sobre su papel como futuro profesional. De estos aprendizajes cabe destacar su gran potencialidad para el desarrollo de las capacidades reflexivas, críticas y el pensamiento de alto nivel en la línea de lo señalado por Díaz Barriga (2003). Integrar la práctica en situaciones profesionales reales obliga al alumnado a tomar decisiones sobre diferentes enfoques posibles de la teoría, variedades metodológicas, opciones tecnológicas, etc. Se destaca la importancia, en el caso de la enseñanza online, de tomar decisiones sobre la selección de la tecnología más adecuada para conseguir objetivos frente a opciones instrumentales acríticas guiadas por la simple presencia de la tecnología (Fueyo y Hevia, 2017).

6. Por otro lado, se ponen en juego habilidades de comunicación concretas que los descentran de su papel de receptores de información y de meros aprendices para ponerles en condición de profesionales que tienen que moderar, dinamizar y dar feedback a un grupo. Se considera que estas habilidades se desarrollan mediante un tipo de interacciones colaborativas que les dan gran relevancia cultural por lo que podrán transferirse a otras situaciones distintas a las originales tal y como señala Díaz-Barriga (2006).

En cuanto a la experiencia de aprendizaje entre iguales que desarrolla el alumnado de $1^{\circ}$ curso, se potencia su doble dimensión de aprendices del curso en cuestión y de analistas de su estructura didáctica, lo que les permite asimilar mejor los contenidos de estudio y ver una aplicación práctica de los mismos. Se favorece, además, la motivación por el aprendizaje, quienes les están instruyendo son compañeros suyos ante los que se sienten con mayor libertad para interaccionar y cuestionar.

Finalmente, resaltar que la experiencia ha contribuido a desarrollar de forma importante diferentes modalidades de competencia digital del alumnado. No solo en lo referido a las competencias para creación de textos y materiales sino también a la implementación de herramientas adecuadas para la enseñanza y el aprendizaje online. Es evidente que se aprovechan los conocimientos previos y el manejo de herramientas tecnológicas que ponen al servicio del desarrollo de competencias vinculadas a su desarrollo profesional y a su aprendizaje mediante estrategias bien definidas, no dejándolas a su propia formación autodidacta como señalan algunos estudios (Espuny, González, Gisbert, 2010). Por último, se considera importante la experimentación que han llevado a cabo ambos grupos en relación con experiencias de formación virtual que hoy por hoy se configuran como alternativas a la formación presencial y que desde el punto de vista de su futura inserción laboral son sumamente relevantes.

\section{Referencias Bibliográficas}

Bardin, L. (1986). L'analyse de contenu. Paris: PUF.

Barrera-Corominas, A., Fernández-de-Álava, M., y Gairín S. J. (2014). Aprendizaje colaborativo en comunidades de práctica online: La Plataforma e-Catalunya. EDUTEC, Revista Electrónica de Tecnología Educativa, 47. Recuperado de http://www. edutec.es/revista/index.php/edutec-e/article/view/118

Barrio, F., y García, F. (2011). Aprendizaje situado y cooperativo en educación superior: una experiencia con equipos de trabajo en Ciencias de la Comunicación. Revista Linhas, 12(2), 18-30.

Bartolomé, A.R., y Steffens, K. (2015). ¿Son los MOOC una alternativa al aprendizaje? Comunicar, 44, 91-99.

Benavides, D., Madrigal, V., y Quiroz, A.P. (2009). La enseñanza situada como herramienta para el logro de un aprendizaje significativo. Centro de Documentación sobre Educación. Recuperado de https://goo.gl/a1ACJA

Bereiter, C. (1997). Situated cognition and how to overcome it. En D. Kirshner y J. A. Whitson (Eds.), Situated cognition. Social, semiotic and psychological perspectives (pp. 281-300). Mahwah, NJ: Lawrence Erlbaum.

Boud, D., Cohen, R., y Sampson, J. (Eds.) (2001). Peer learning in higher education. Londres: Kogan.

Brown, J., Collins, A., y Duguid, P. (1989). Situated cognition and the culture of learning. Educational Researcher, 18 (1), 32-42.

Cela-Ranilla, J., Esteve, V., Esteve, F., González, J., y Gisbert-Cervera, M. (2017). El docente en la sociedad digital: una propuesta basada en la pedagogía transformativa y en la tecnología avanzada. Profesorado. Revista de Currículum y Formación de Profesorado, 21(1), 403-422.

Cobo, C., y Moravec, J.W. (2011). Aprendizaje invisible. Hacia una nueva ecología de la educación. Barcelona: Publicacions i Edicións de la Universitat de Barcelona.

Daniel, J. (2012). Making Sense of MOOCs: Musings in a Maze of Myth, Paradox and Possibility. Seoul: Korean National Open University.

Davies, C., Hart, A., Eryigit-Madzwamuse, S., Stubbs, C., Aumann, K., Aranda, K., y Heaver, B. (2017). Communities of Practice in Community-University Engagement: Supporting Co-Productive Resilience Research and Practice. En J. McDonald y A. Cater-Steel (Eds.), Communities of Practice (pp. 175-198). Singapore: Springer Singapore.

Díaz-Barriga, F. (2006). Enseñanza situada: vínculo entre la escuela y la vida. México: Mc Graw-Hill.

Díaz-Barriga, F. (2003). Cognición situada y estrategias para el aprendizaje significativo. Revista electrónica de investigación educativa, 5(2), 105-117.

Enguita, M. (2013). El aprendizaje difuso y el declive de la institución escolar. Revista de la Asociación de Sociología de la Educación (RASE), 6(2), 150-167. 
Espuny, C., González, J., y Gisbert, M. (2010). ¿Cuál es la competencia digital del alumnado al llegar a la universidad? Datos de una evaluación cero. Enseñanza \& Teaching, 28, 113-137.

Fernández, E., y Anguita, R. (2015). Aprendizajes invisibles en contextos de educación expandida. Retos y oportunidades en la sociedad hiperconectada. Profesorado. Revista de Currículum y Formación de Profesorado, 19(2), 1-16.

Fischer, G., Rohde, M., y Wulf, V. (2007). Community-based learning: The core competency of residential, research-based universities. International Journal of Computer-Supported Collaborative Learning (IJCSCL), 2(1), 9-40.

Fueyo, A., y Hevia, I. (2017). Network Learning through Communities of Inquiry on massive online learning environments. Digital Education Review, 31, 116-130.

Gil-Izquierdo, M., Fernández-Pinedo, N., y Pérez-Encinas, A. (2017). Creation of teaching Communities (CoP) and Networks of Practice (NoP) for enhancing active learning in the University context. MPRA Munich Personal RePEc Archive, 77017. Recuperado de https://mpra.ub.uni-muenchen.de/77017/

Gisbert, M., y Esteve, F. (2011). Digital Leaners: la competencia digital de los estudiantes universitarios. La cuestión universitaria, 7, 48-59.

González, N., y García, J.L. (2012). Metodologías participativas para la mejora del aprendizaje en educación superior. Un proyecto innovador con estudiantes de la facultad de Educación. Revista Iberoamericana para la Investigación y el Desarrollo Educativo, 3(5), 80-93.

Hernández-Sellés, N., González-Sanmamed, M., y Muñoz-Carril, P.C. (2015). El rol docente en las ecologías de aprendizaje: análisis de una experiencia de aprendizaje colaborativo en entornos virtuales. Profesorado. Revista de Currículum y Formación de Profesorado, 19(2), 147-163.

Jordan, K. (2014). Initial trends in enrolment and completion of massive open online courses Massive Open Online Courses. International Review of Research in Open and Distance Learning, 15(1).
Lave, J. (1997). The culture of acquisition and the practice of understanding. En D. Kirshner y J. A. Whitson (Eds.), Situated cognition. Social, semiotic and psychological perspectives (pp. 1735). Mahwah, NJ: Lawrence Erlbaum.

Lave J., y Wenger E. (1991). Situated Learning: Legitimate Peripheral Participation. Cambridge UK: Cambridge University Press.

Leguízamo, A., Montaño, N., y Villarroel, G. A. (2004). Una Experiencia de Aprendizaje Situado: Laboratorio de Desarrollo de Software. EDUTEC 2004 Barcelona. Recuperado de http://www. lmi.ub.es/edutec2004/pdf/156.pdf

McDonald, J., y Cater-Steel, A. (Eds.) (2016). Communities of Practice: Facilitating Social Learning in Higher Education. Singapore: Springer Singapore.

Mezirow, J. (2000). Learning as Transformation. Critical Perspectives on a Theory in Progress. San Francisco: Jossey-Bass.

Niemeyer, B. (2006). El aprendizaje situado: una oportunidad para escapar del enfoque del déficit. Revista de Educación, 341, 99-121.

Osuna-Alcedo, S., Marta-Lazo-C., y Frau-Meigs, D. (2018). De sMOOC a tMOOC, el aprendizaje hacia la transferencia profesional: El proyecto europeo ECO. Comunicar, 55, 105-114.

Paz, H. (2007). El aprendizaje situado como una alternativa en la formación de competencias en ingeniería. Revista Educación en Ingeniería, 4, 1-13.

Reaburn, P., y McDonald, J. (2016). Creating and Facilitating Communities of Practice in Higher Education: Theory to Practice in a Regional Australian University. En J. McDonald y A. Cater-Steel (Eds.), Communities of Practice: Facilitating Social Learning in Higher Education (pp. 121-150). Singapore: Springer Singapore

Rodríguez, R. (2006). Diseño de entornos para el desarrollo de la autonomía en el aprendizaje. Aula Abierta, 87, 89-104.

Taylor, E.W. (2008). Transformative Learning Theory. New Directions for Adult and Continuing Education, 119, 5-15.

Vygotsky, L. (1986). Pensamiento y lenguaje. Buenos Aires: La Pléyade.

Wenger, E. (2001). Comunidades de práctica, aprendizaje, significado $e$ identidad. Barcelona: Paidós. 